\title{
ELEMENTARY AMENABLE GROUPS OF FINITE HIRSCH LENGTH ARE LOCALLY-FINITE BY VIRTUALLY-SOLVABLE
}

\author{
J. A. HILLMAN and P. A. LINNELL
}

(Received 26 March 1990)

Comimunicated by $\mathbf{H}$. Lausch

\begin{abstract}
If $G$ is an elementary amenable group of finite Hirsch length $h$, then the quotient of $G$ by its maximal locally finite normal subgroup has a maximal solvable normal subgroup, of derived length and index bounded in terms of $h$.

1991 Mathematics subject classification (Amer. Math. Soc.): Primary 20 F 19; Secondary 20 F 38.

Keywords and phrases: cohomological dimension, elementary amenable, group, Hirsch length, solvable.
\end{abstract}

The class of elementary amenable groups is the class of groups generated from the finite groups and $\mathbb{Z}$ by the operations of extension and increasing union. This class arose first in connection with the Banach-Tarski paradox [6] and more recently has been of interest to topologists as the largest class of groups over which topological surgery techniques are known to work in dimension 4 [2]. It clearly contains all locally finite groups and solvable groups, and hence contains all locally finite by virtually solvable groups. In [3] the notion of Hirsch length (as a measure of the size of a solvable group) was extended to this class and it was shown that elementary amenable groups of Hirsch length at most 3 are locally finite by solvable while in general the Hirsch length is bounded above by the rational cohomological dimension. Here we shall show that every elementary amenable group of finite Hirsch length is locally finite by virtually solvable. In particular, if a group of finite cohomological

(C) 1992 Australian Mathematical Society 0263-6115/92 \$A2.00+0.00 
dimension has a nontrivial elementary amenable normal subgroup then it has a nontrivial abelian normal subgroup. (This answers a question raised in [5] in connection with extending a theorem of Gottlieb).

We recall briefly the description of the class of elementary amenable groups given in [4] and the definition of Hirsch length given in [3]. If $\mathscr{Z}$ and $\mathscr{Y}$ are classes of groups let $\mathscr{X} \mathscr{Y}$ denote the class of $(\mathscr{Z}$ by $\mathscr{Y}$ ) groups $G$ which have a normal subgroup $H$ in $\mathscr{Z}$ such that the quotient $G / H$ is in $\mathscr{Y}$, and let $L \mathscr{Z}$ denote the class of groups such that each finitely generated subgroup is contained in some $\mathscr{X}$-subgroup. Let $\mathscr{X}_{0}=\{1\}$ and let $\mathscr{X}_{1}$ be the class of finitely generated abelian by finite groups. If $\mathscr{X}_{\alpha}$ has been defined for some ordinal $\alpha$ let $\mathscr{X}_{\alpha+1}=\left(L \mathscr{X}_{\alpha}\right) \mathscr{X}_{1}$ and if $\mathscr{X}_{\alpha}$ has been defined for all ordinals $\alpha$ less than some limit ordinal $\beta$ let $\mathscr{X}_{\beta}=\bigcup_{\alpha<\beta} \mathscr{Z}_{\alpha}$. Then the class of elementary amenable groups is $\bigcup \mathscr{X}_{\alpha}$, where the union is taken over all ordinals. (In fact, it is not hard to show by transfinite induction on $\alpha$ that if $\kappa$ is the first uncountable ordinal then every countable group in $\mathscr{Z}_{\alpha}$ is in $\mathscr{X}_{\kappa}$. Hence every elementary amenable group is in $\left.\mathscr{X}_{\kappa+1}\right)$.

The Hirsch length function $h$ takes values in $\mathbb{Z}_{\geq 0} \cup\{\infty\}$, where $\mathbb{Z}_{\geq 0}$ is the set of nonnegative integers. If $G$ is in $\mathscr{Z}_{1}$ let $h(G)$ be the rank of an abelian subgroup of finite index in $G$. If $h(G)$ has been defined for all groups $G$ in $\mathscr{X}_{\alpha}$ and $H$ is in $L \mathscr{X}_{\alpha}$ let $h(H)=1$. u.b. $\left\{h(F) \mid F\right.$ is an $\mathscr{X}_{\alpha}$ subgroup of $H\}$. Finally, if $G$ is in $\mathscr{Z}_{\alpha+1}$, so has a normal subgroup $H$ in $L \mathscr{X}_{\alpha}$ with quotient in $\mathscr{X}_{1}$, let $h(G)=h(H)+h(G / H)$. Transfinite induction on $\alpha(G)=\min \left\{\alpha \mid G\right.$ is in $\left.\mathscr{Z}_{\alpha}\right\}$ may be used to prove (simultaneously) that $h(G)$ is well defined, that if $H$ is a subgroup of $G$ then $h(H) \leq h(G)$, that if $H$ is a normal subgroup of $G$ then $h(G)=h(H)+h(G / H)$ and that $h(G)=1$. u. b. $\{h(F) \mid F$ is a finitely generated subgroup of $G\}$.

Let $\mathscr{F}$ be the class of finite groups and $\mathscr{S}$ the class of solvable groups; $L F$ is then the class of locally finite groups and $\mathscr{F}$ is the class of virtually solvable groups. Every group $G$ has a unique maximal normal $L \mathscr{F}$-subgroup $\Lambda(G)$. Although in general an infinite group need not have a maximal solvable normal subgroup this is so if it is an $\mathscr{S} F$-group. Similarly if a group has a normal $(L \mathscr{F}) \mathscr{S}$-subgroup of finite index then it has a maximal normal $(L \mathscr{F}) \mathscr{S}$-subgroup, necessarily of finite index. (This follows from the facts that the product of two normal subgroups is again a normal subgroup, and that if the subgroups are each in $(L \mathscr{F}) \mathscr{S}$ so is their product). Our main result shall follow by induction on $h$ after making careful estimates of the index and derived length modulo $\Lambda$ of such maximal normal $(L \mathscr{F}) \mathscr{S}$. subgroups, as functions of $h$.

THEOREM. There are functions $d$ and $M$ from $\mathbb{Z}_{\geq 0}$ to $\mathbb{Z}_{\geq 0}$ such that if $G$ is an elementary amenable group of Hirsch length at most $h$ and $\Lambda(G)$ 
is it maximal locally finite normal subgroup then $G / \Lambda(G)$ has a maximal solvable normal subgroup of derived length at most $d(h)$ and index at most $M(h)$.

Proof. We argue by induction on $h$. Since an elementary amenable group has Hirsch length 0 if and only if it is locally finite we may set $d(0)=0$ and $M(0)=1$. Assume that the result is true for all such groups with Hirsch length at most $h$ and that $G$ is an elementary amenable group with $h(G)=h+1$.

Suppose first that $G$ is finitely generated. Then by [3, Lemma 1] there are normal subgroups $K<H$ in $G$ such that $G / H$ is finite, $H / K$ is free abelian of rank $r \geq 1$ and the action of $G / H$ on $H / K$ by conjugation is effective. (Note that $r=h(G / K) \leq h(G)=h+1)$. Since the kernel of the natural map from $G L(r, \mathbb{Z})$ to $G L(r, \mathbb{Z} / 3 \mathbb{Z})$ is torsion free, we see that $G / H$ embeds in $\mathrm{GL}(r, \mathbb{Z} / 3 \mathbb{Z})$ and so has order at most $3^{r^{2}}$. Since $h(K)=h(G)-r \leq h$ the inductive hypothesis applies for $K$, so it has a normal subgroup $L$ containing $\Lambda(K)$ and of index at most $M(h)$ such that $L / \Lambda(K)$ has derived length at most $d(h)$ and is the maximal solvable normal subgroup of $K / \Lambda(K)$. As $\Lambda(K)$ and $L$ are characteristic in $K$ they are normal in $G$. (In particular, $\Lambda(K)=K \cap \Lambda(G))$. The centralizer of $K / L$ in $H / L$ is a normal solvable subgroup of $G / L$ with index at most $[K: L] ![G: H]$ and derived length at most 2. Set $M^{\prime}=M(h) ! 3^{(h+1)^{2}}$ and $d^{\prime}=M^{\prime}+2+d(h)$. Then $M^{\prime}$ and $d^{\prime}$ depend only on $h$, and $G / \Lambda(G)$ has a maximal solvable normal subgroup of index at most $M^{\prime}$ and derived length at most $d^{\prime}$ (since it contains the preimage of the centralizer of $K / L$ in $H / L)$.

In general let $\left\{G_{i} \mid i\right.$ in $\left.I\right\}$ be the set of finitely generated subgroups of $G$. Since $G_{i}$ is a finitely generated elementary amenable group with Hirsch length at most $h+1$ we see from the previous paragraph that $G_{i}$ has a normal subgroup $H_{i}$ containing $\Lambda\left(G_{i}\right)$ such that $H_{i} / \Lambda\left(G_{i}\right)$ is the maximal solvable normal subgroup of $G_{i} / \Lambda\left(G_{i}\right)$ and has derived length at most $d^{\prime}$ and index at most $M^{\prime}$. Clearly $H_{i}$ is the maximal normal $(L \mathscr{F}) \mathscr{S}$-subgroup of $G_{i}$. Write $N=\max \left\{\left[G_{i}: H_{i}\right] \mid i\right.$ in $\left.I\right\}$ and choose $\alpha$ in $I$ such that $\left[G_{\alpha}: H_{\alpha}\right]=N$. Now if $G_{i} \geq G_{\alpha}$ then $H_{i} \cap G_{\alpha}$ is a normal $(L \mathscr{F}) \mathscr{S}_{\text {- }}$ subgroup of $G_{\alpha}$ and so $H_{i} \cap G_{\alpha} \leq H_{\alpha}$. Therefore

$$
N=\left[G_{\alpha}: H_{\alpha}\right] \leq\left[G_{\alpha}: H_{i} \cap G_{\alpha}\right]=\left[H_{i} G_{\alpha}: H_{i}\right] \leq\left[G_{i}: H_{i}\right] \leq N,
$$

and so $\left[G_{i}: H_{i}\right]=N$ and $H_{i} \geq H_{\alpha}$. It is now easy to see that if $G_{i} \geq G_{j} \geq G_{\alpha}$ then $H_{i} \geq H_{j}$.

Set $J=\left\{i\right.$ in $\left.I \mid H_{i} \geq H_{\alpha}\right\}$ and $H=\bigcup_{i \in J} H_{i}$. If $x$ and $y$ are in $H$ and $g$ is in $G$ then there are indices $i, j$ and $k$ in $J$ such that $x$ is in 
$H_{i}, y$ is in $H_{j}$ and $g$ is in $G_{k}$. Choose $l$ in $J$ such that $G_{l}$ contains $G_{i} \cup G_{j} \cup G_{k}$. Then $x$ and $y$ are in $H_{l}$ by the previous paragraph, so $x y^{-1}$ and $\mathrm{gxg}^{-1}$ are in $H_{l}$. Hence $H$ is a normal subgroup of $G$. Moreover, if $x_{1}, \ldots, x_{N}$ is a set of coset representatives for $H_{\alpha}$ in $G_{\alpha}$ then $x_{1}, \ldots, x_{N}$ is also a set of coset representatives for $H$ in $G$, and so $[G: H]=N$.

Write $D_{i}$ for the $d^{\prime}$ derived subgroup of $H_{i}$. Then $D_{i}$ is a locally finite normal subgroup of $G_{i}$, and so by an argument similar to that of the previous paragraph $\bigcup_{i \in J} D_{i}$ is a locally finite normal subgroup of $G$. Since it is easily seen that the $d^{\prime}$ derived subgroup of $H$ is contained in $\bigcup_{i \in J} D_{i}$ (as each iterated commutator involves only finitely many elements of $H$ ) it follows that $H \Lambda(G) / \Lambda(G) \approx H / H \cap \Lambda(G)$ is solvable and of derived length at most $d^{\prime}$. Thus the proof is completed by setting $d(h+1)=d^{\prime}$ and $M(h+1)=M^{\prime}$.

Corollary 1. If $G$ is an elementary amenable group such that $h(G)$ is finite and which has no nontrivial locally finite normal subgroup then $G$ is virtually solvable, and hence is countable.

Proof. The virtual solvability follows immediately from the theorem, and the countability then follows from [1, Lemma 7.9].

COROllary 2. A group $G$ of finite cohomological dimension which has a nontrivial elementary amenable normal subgroup has a nontrivial (torsion free) abelian normal subgroup.

Proof. If $H$ is such an elementary amenable normal subgroup then it has finite cohomological dimension and so $h(H)<\infty$ by [3, Lemma 2]. Moreover $H$ is torsion free, so by the theorem it is virtually solvable. The lowest nontrivial member of the derived series for the maximal solvable normal subgroup of $H$ is abelian, and as it is characteristic in $H$ it is normal in $G$.

The second corollary implies that the apparent increase in generality of [5, Theorem 3] over Rosset's version of Gottlieb's theorem (that if $X$ is an aspherical finite complex and $\pi_{1}(X)$ has a nontrivial abelian normal subgroup then $\chi(X)=0$ ) is illusory. However the main result of [5] is needed to prove the converse, stated in [3], that if $X$ is a $[G, m]$-complex with $\chi(X)=0$ and $G=\pi_{1}(X)$ has a nontrivial torsion free elementary amenable normal subgroup then $X$ is aspherical.

We conclude with a question. Is every group of finite cohomological dimension and which has no noncyclic free subgroup virtually solvable? 


\section{References}

[1] R. Bieri, Homological dimension of discrete groups (Queen Mary College Mathematics Notes, London, 1976).

[2] M. H. Freedman, 'The disk theorem for four-dimensional manifolds,' Proc. Internat. Cong. Math., vol. 1, pp. 647-663 (Warsaw, 1983).

[3] J. A. Hillman, 'Elementary amenable groups and 4-manifolds with Euler characteristic 0,' J. Austral. Math. Soc. Ser. A 50 (1991), 160-170.

[4] P. H. Kropholler, P. A. Linnell, and J. A. Moody, 'Applications of a new $K$-theoretic theorem to soluble group rings,' Proc. Amer. Math. Soc. 104 (1988), 675-684.

[5] P. A. Linnell, 'Zero divisors and group von Neumann algebras,' Pacific J. Math. 149 (1991), 349-363.

[6] J. von Neumann, 'Zur allgemeine Theorie der Masse,' Fund. Math. 13 (1929), 73-116.

The University of Sydney

Sydney

NSW 2006 Australia
Virginia Polytechnic Institute and State University Blacksburg, VA 24061-0123 